\title{
Magnetic resonance imaging evaluation of perianal fistulas
}

${ }^{1}$ Pozitron Medical Investigation

${ }^{2}$ Faculty of Medicine, University "Ovidius" of Constanta

\section{ABSTRACT}

MRI has become the method of choice for evaluating perianal fistulae due to its ability to display the anatomy of the sphincter muscles orthogonally, with good contrast resolution. In this article we give an outline of the classification of perianal fistulae and present a pictorial assay of sphincter anatomy and the MRI findings in perianal fistulae. This study is based on a retrospective analysis of 21 patients with a clinical diagnosis of perianal fistula.

Keywords: fistula, perianal, MRI

\section{Deacu Cristina Madalina}

Bd. Ferdinand 108, bl. R4, sc. B, ap. 17

Constanta, Romania

e-mail: cristina.deacu@yahoo.com

phone: +40723649000

\section{Introduction}

\section{Definition}

Perianal fistula is an abnormal connection between the anal canal and the skin of the perineum, established secondary to the perirectal tissue inflammatory process.

History

Anal fistulas were known from the time of Hippocrates, but they began to receive special attention in the 19th century, when Frederick Salmon founded the Benevolent Dispensary for the Relief of the Poor Afflicted with Fistula, Piles, and Other Diseases of the Rectum and Lower Intestines. Later, Goodsall described the course of fistulous tracks from the skin to the anus and Parks made a classification of fistulas widely used in surgical practice [1].

\section{Anatomy}

Knowledge of the anatomy of the anal sphincter complex and surrounding spaces is very important for 
image interpretation (Figure 1 and 2).

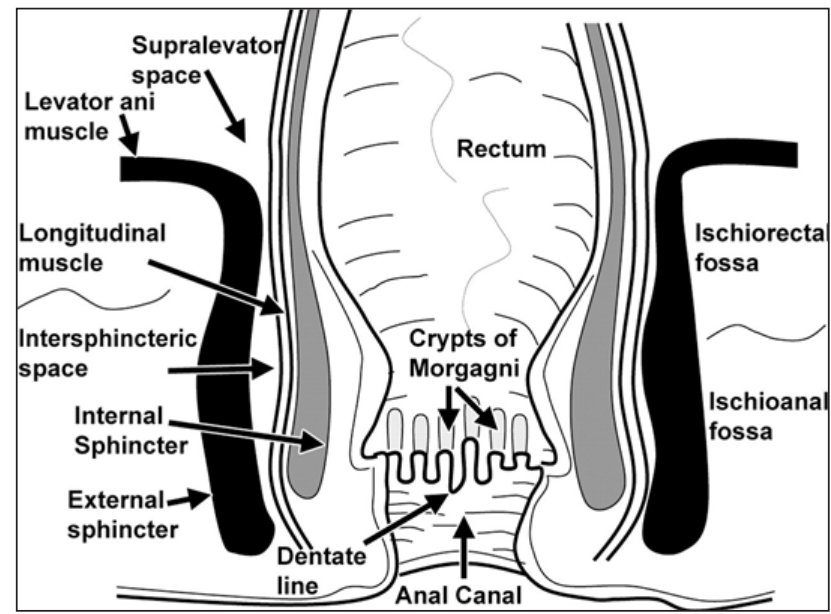

Figure 1. Normal anatomy of the anal canal in the coronal plane. RadioGraphics 2012 [5]

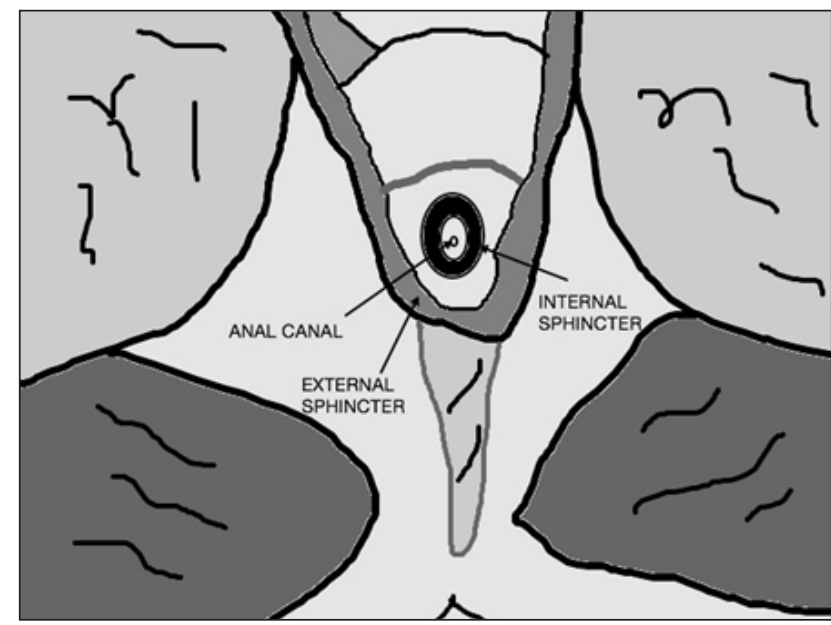

Figure 2. Normal anatomy of the anal canal in axial plane

The anal canal is the terminal part of the large intestine. It is situated between the rectum and anus, below the level of the pelvic diaphragm. It lies in the anal triangle of perineum in between the right and left ischioanal fossa. It is approximately $2.5-4 \mathrm{~cm}$ long, extending from the anorectal junction to the anus. It is directed downwards and backwards. It is surrounded by inner involuntary and outer voluntary sphincters which keep the lumen closed in the form of an anteroposterior slit [2].

The anatomy of the perianal region is well demonstrated on coronal (figure 3) and axial (figure 4) MR images.

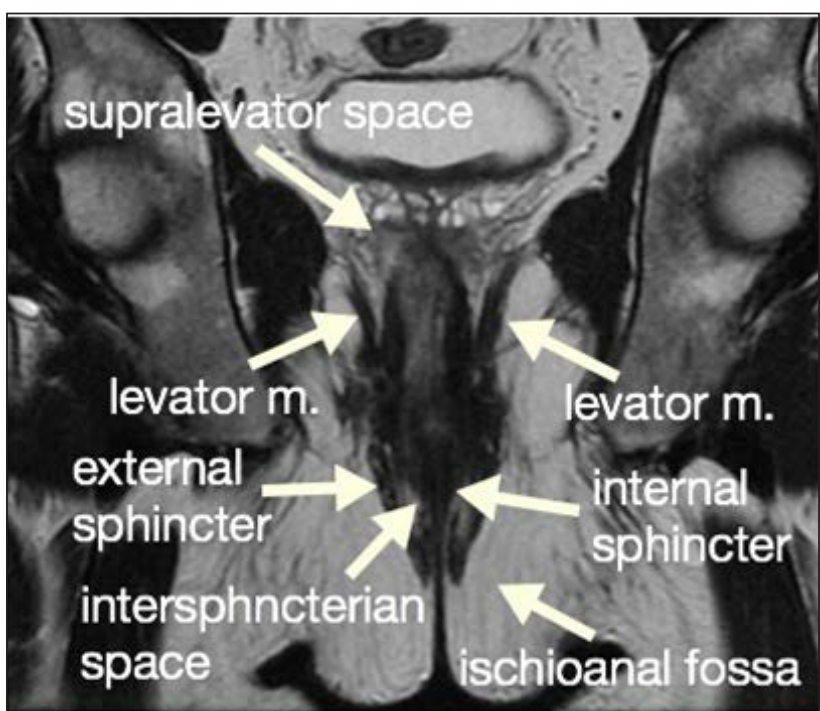

Figure 3. Coronal T2-weighted image. Anatomy

The coronal images depict the levator ani muscle (levator plane), the identification of which is important to distinguish supralevator from infralevator infections. The puborectalis ring is seen as a thickening of the superior fibers of the external sphincter. The puborectalis further merges with the levator plate superiorly.

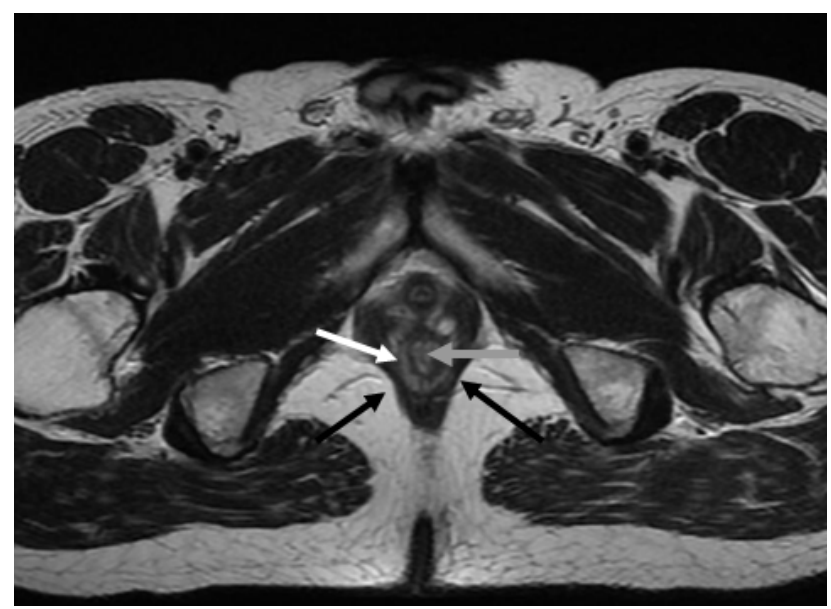

Figure 4. Axial T2-weighted image. Black arrow external sphincter, white arrow - internal sphincter, grey arrow - anal cana 
The external anal sphincter (a striated muscle) is clearly visualized on MRI. It is hypointense on $\mathrm{T} 1 \mathrm{~W}$ and $\mathrm{T} 2 \mathrm{~W}$ images and is bordered laterally by the fat in the ischioanal fossa.

The internal sphincter (a smooth muscle) is hypointense on T1W and T2W TSE images and is relatively hyperintense on fat-suppressed $\mathrm{T} 2 \mathrm{~W}$ images. It shows enhancement on post-gadolinium T1W images (Figure 5).

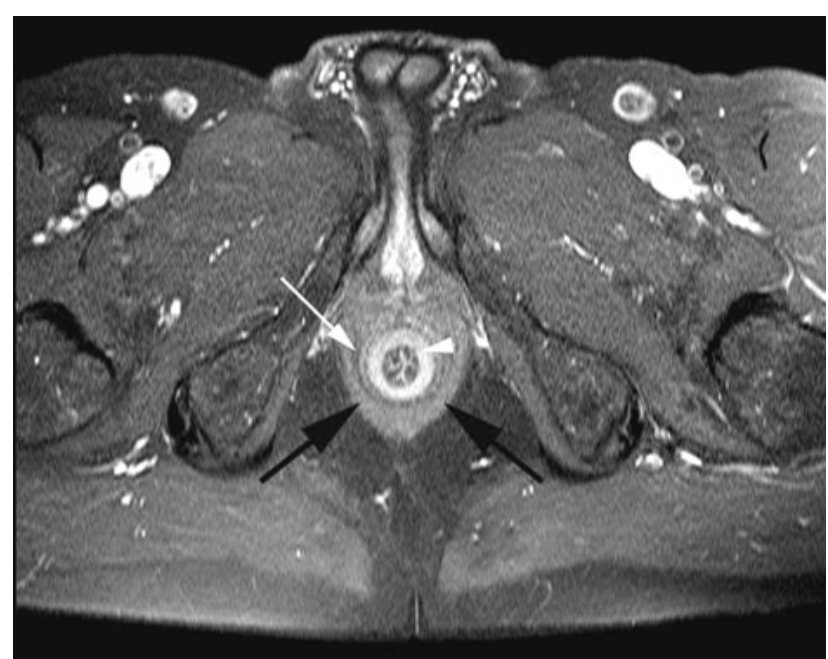

Figure 5. Axial T1 fat saturated post-gadolinium. Black arrow - external sphincter, white arrow - internal sphincter, white arrow head - anal canal

\section{Material and methods}

The present study included 21 patients (16 males and 5 females; with age range 38-62 years; mean age 50 years) with perianal sepsis in the period between January 2010 and July 2015. Those patients were referred to Pozitron Medical Investigation as they had clinical manifestation of suspected perianal sepsis and a visible external opening.

MRI Imaging protocol

There is no need for prior special preparation of the patient. We are using a Siemens Magnetom Symphony and GE Optima360 Advance 1.5T MRI scanners. Patient lies in supine position, with arms by his side. A phased array 32 channel body coil is used. Protocol includes: T1-weighted fast spin echo in axial plane (planed parallel to the pelvic diaphragm) and T2-weighted fast spin echo in all plans (for highlighting muscle groups and fistulous tract; coronal is planed parallel to the anal canal), T2-weighted with fat suppression images in axial and coronal planes (for better visualization of active fistulous tracts, oedematous changes and abscess) and diffusion weighted images (to confirm active fistulous tracts and abscesses). After obtaining this images, contrast material (Gd DPTA - Magnevist) is injected IV with a dose of $0.1 \mathrm{mmol} / \mathrm{kg}$. Then T1-weighted with fat suppression images are obtained (assessing the presence and extent of inflammation).

\section{Resulits}

Twenty-one patients with perianal sepsis were included in this study, 4 cases grade 1 (simple linear intersphincteric fistula) (Fig. 6), 4 cases grade 2 (intersphincteric fistula with abscess or secondary track) (Figure 7), 7 cases grade 3 (trans-sphincteric fistula) (Figure 8), 5 cases grade 4 (trans-sphincteric fistula with abscess (3 cases), secondary track within the ischiorectal fossa (1 case) and both (1 case)) (Figure 9) and 1 case grade 5 (supralevator) (Figure $10)$.

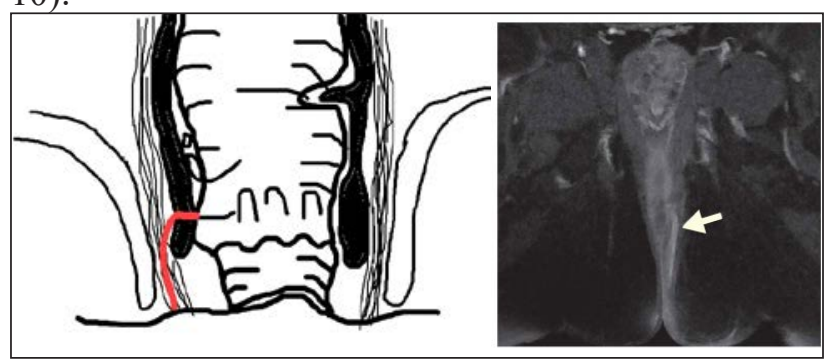

FIgure 6. Grade I fistula

Simple trans-sphincteric fistula and transsphincteric fistula complicated by abscess or secondary track were the most frequent findings. 
MRI results were in good agreement with the surgical findings.

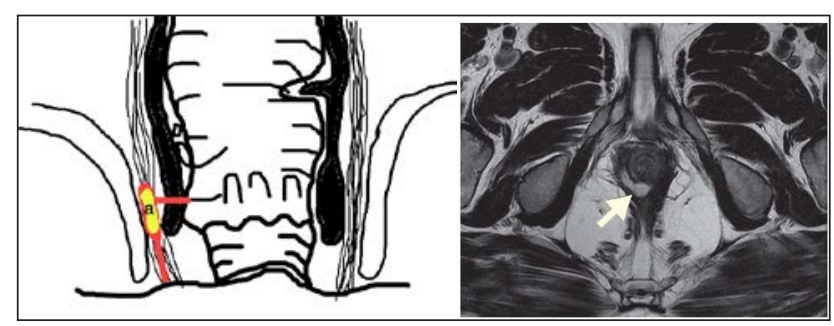

Figure 7. Grade II fistula

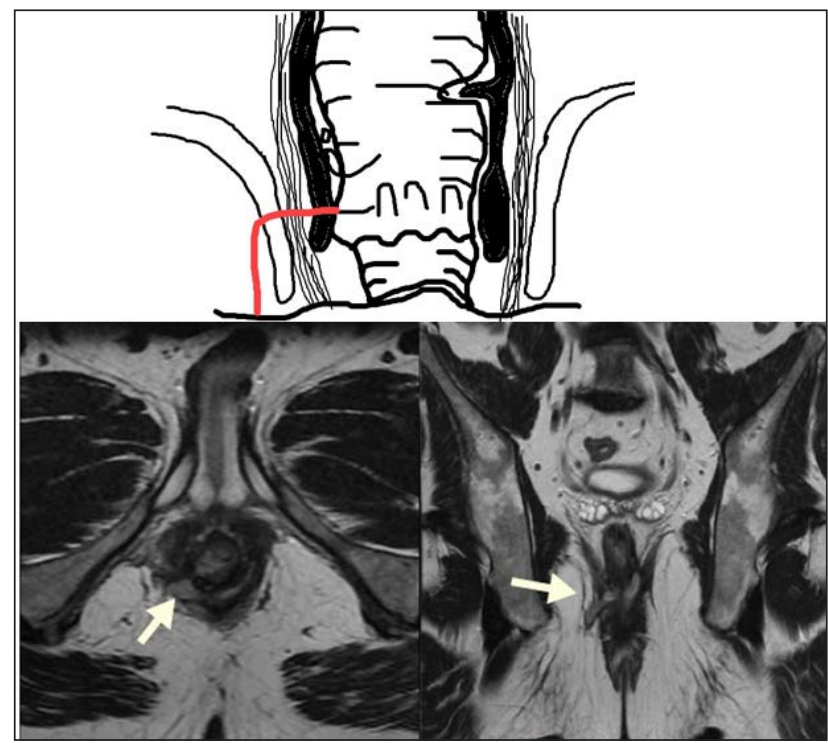

Figure 8. Grade III fistula

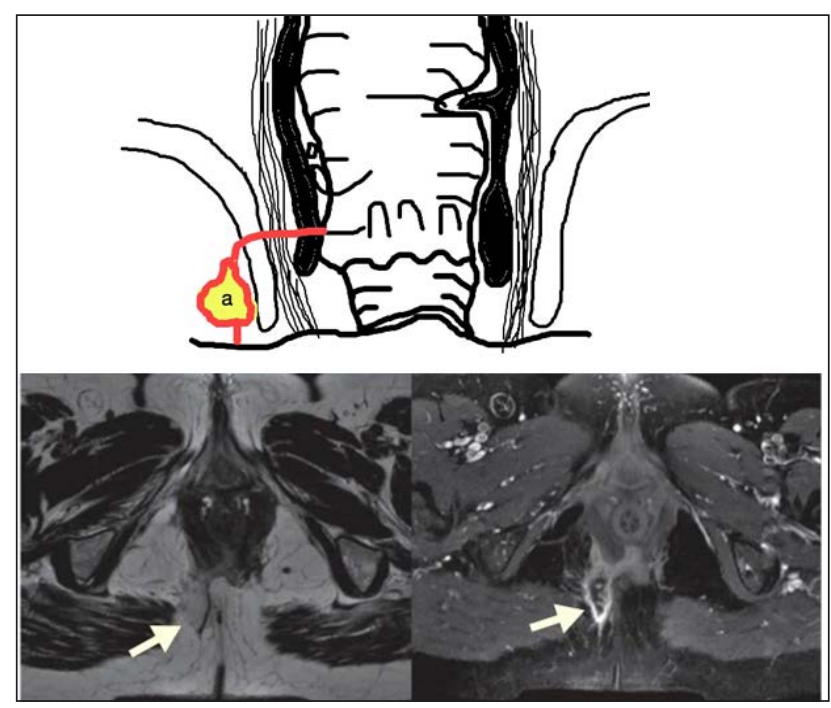

Figure 9. Grade IV fistula

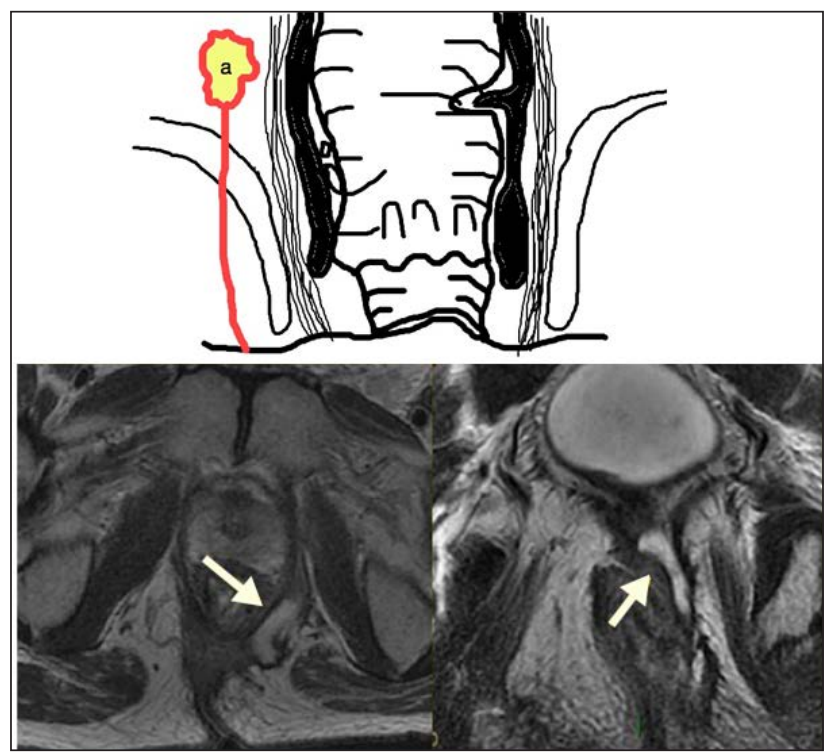

Figure 10. Grade V fistula

The sensitivity of MRI in the detection of perianal fistula was, in our case, $100 \%$.

Four cases were active fistulas and seventeen were inactive ones.

Seventeen fistulas arose from at the dentate line posteriorly.

Perianal abscesses may occur anywhere along a fistula tract and typically have central hyperintense signal on T2-weighted imaging corresponding to pus with peripheral rim enhancement secondary to the fibrous wall and surrounding inflammation. We had 3 cases with perianal abscess (Figure 11)

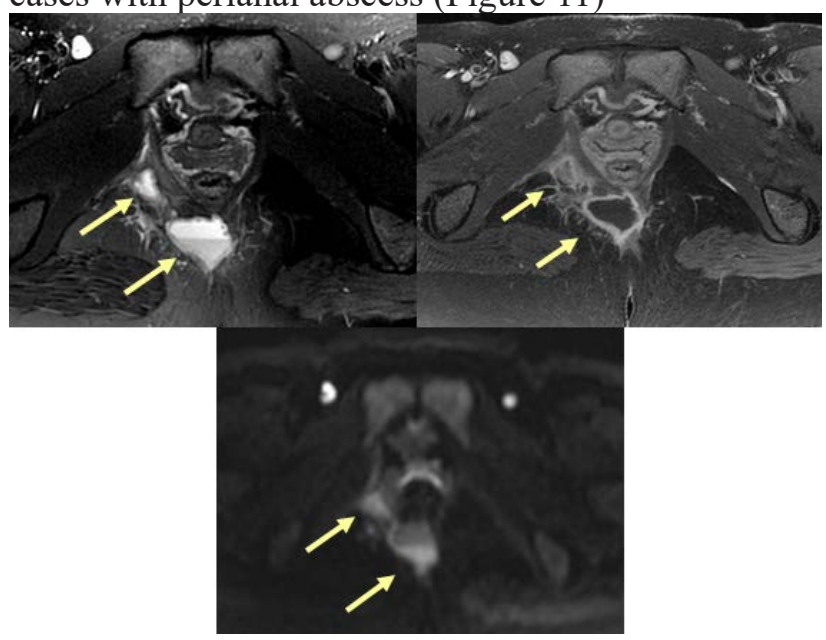

Figure 11. Abcess. T2-weighted image, T1-FS + Gd, diffusion weighted image 


\section{Discussion}

Perianal fistulas are classified according to the St James's University Hospital using simple anatomical landmarks, allowing radiologists to provide surgeons the necessary data to establish an appropriate treatment:

Grade 1: simple linear inter-sphincteric fistula.

Grade 2: intersphincteric fistula with abscess or secondary track.

Grade 1 and 2 need simple surgical treatment.

Grade 3: trans-sphincteric fistula.

Grade 4: trans-sphincteric fistula with abscess or secondary track within the ischiorectal fossa.

Grade 3 and 4 need complex surgical treatment (+/- continence +/- colostoma).

Grade 5: supralevator and translevator disease (needs searching for septic pelvic source) [1].

The purpose of MRI is to find the fistulous trajectory topography in order to classify them, to analyze the presence and topography of abscesses or secondary tracts, to see the location of internal orifices (intraluminal) and external (usually the skin) and the activity of fistula and also, to see the anorectal wall, sphincters, the neighboring organs and the remote extension.

Once a fistula tract is detected, its relationship to the sphincter complex and location of the internal and external openings should be described. The internal opening can be described according to anteriorposterior and right-left locations or according to the "anal clock" with the patient in the supine position. (Figure 12)

The surgical exploration without previous MRI diagnostics can be made difficult due to the presence of fibrosis and oedema, so that the MRI can identify the hidden intersphincteric space with the captured puss without rupturing the cutaneous layer.

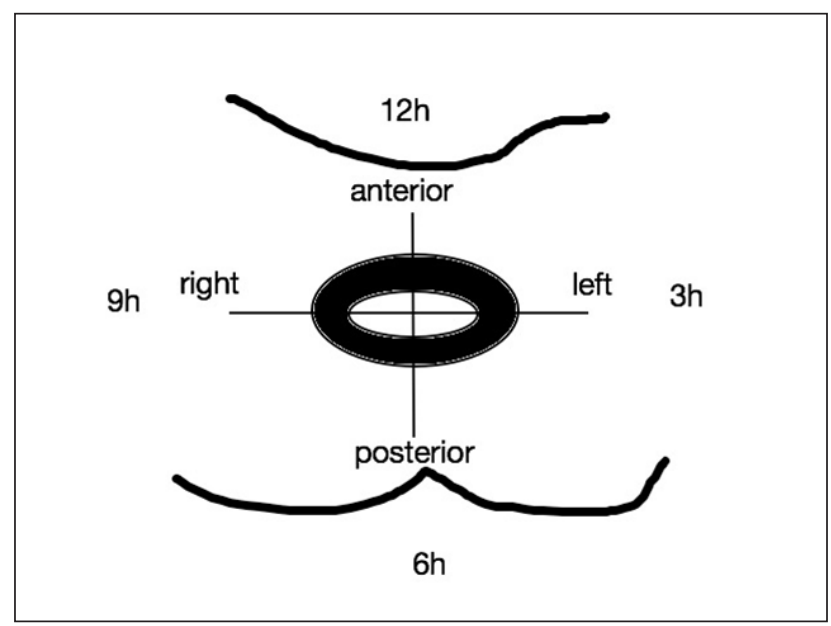

Figure 12. Anal clock

Table I. MRI fistula semiology

\begin{tabular}{|c|c|c|c|}
\hline $\begin{array}{c}\text { Active } \\
\text { fistula }\end{array}$ & $\begin{array}{c}\text { Sequelar } \\
\text { fistula }\end{array}$ & Abcess & \\
\hline T1w & hipo & hipo & hipo \\
\hline T2w & Hiper & hipo & Hiper \\
\hline STIR & Hiper & hipo & Hiper \\
\hline T1w+GD & Enhancement & hipo & $\begin{array}{c}\text { hipo, wall } \\
\text { enhancement }\end{array}$ \\
\hline
\end{tabular}

Active fistula tract appears as a hypointense linear structure on T1-weighted imaging and hyperintense on T2-weighted imaging (best visualized with fat saturation) and enhances with IV contrast agent (Table I). Granulation tissue with increased vascularity is thought to account for the T2-weighted imaging hyperintensity and contrast enhancement. (Figure 13) [3].

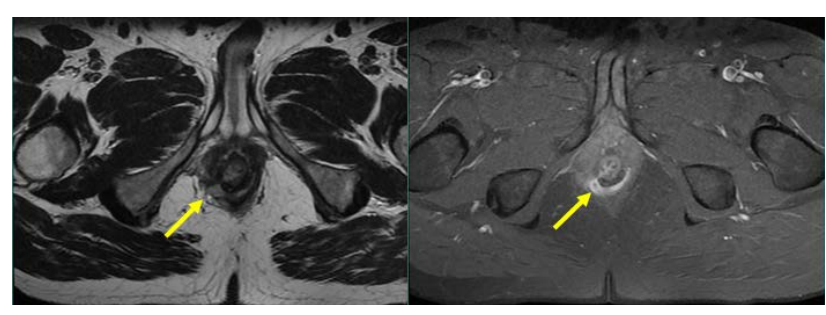

Figure 13. Axial T2 and axial T1 FS $+G d$. Recent fistula

Inactive tracts are also hypointense on T1weighted imaging but lack the associated T2- 
weighted imaging hyperintensity and contrast enhancement. Tissues surrounding the tract may also show hyperintensity on T2-weighted imaging if there is edema or inflammation. (Figure 14).

Loss of T2-weighted imaging hyperintense signal precedes lack of enhancement and proposed a predictable stepwise response to therapy, first as reduced pus production and then as diminished inflammation within the fistula. These stepwise changes also correlated well with clinical response, suggesting that MRI follow-up may be used to guide therapy [4].

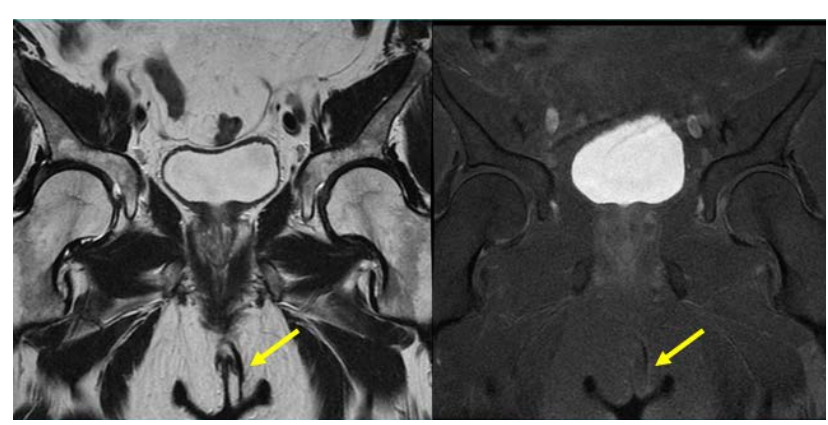

Figure 14. Coronal T2 and coronal T1 FS postgadolinium. Old fistula.

The most common indications for MRI are: recurrent fistulas, following the fistulae treated in two steps for checking the effect of drainage, in case of incomplete clinical data in complex fistulae, in case of anal stenosis (clinical examination impossible), in Crohn's disease, because fistulas in Crohn are complex, high, recurring, with abscesses and often unknown primary orifice.

\section{Conclusions}

MRI is a useful procedure for successful management of peri-anal fistula by correct assessment of the extent of disease and relationship to sphincter complex. Also it helps in the identification of secondary extensions, particularly horseshoe tracts and abscesses resulting in complete evaluation and highest possible diagnostic accuracy aiding successful surgical interventions, aiming to reduce complications and recurrences.

Radiologists should be familiar with the anatomic and pathologic findings of perianal fistulas and classify them using the St James's University Hospital MR imaging-based grading system. In this way, appropriate surgical management can be planned and recurrences can be prevented.

\section{References}

1. Parks, A.G., Gordon \& P.H., Hardcastle, J.D. (1976). A classification of fistula-in-ano. $\mathrm{Br} J$ Surg 63, 1-12.

2. Toyonaga, T., Matsushima, M. \& Tanaka, Y. (2007). Microbiological analysis and endoanal ultrasonography for diagnosis of anal fistula in acute anorectal sepsis, Int $J$ Colorectal Dis. 22, 209-213

3. Halligan, S. \& Stoker, J., (2006). Imaging of fistula in ano. Radiology 239, 18-33.

4. Savoye-Collet, C., Savoye, G., Koning, E., Dacher, J.N. \& Lerebours, E. (2010). Fistulizing perianal Crohn's disease: contrast-enhanced magnetic resonance imaging assessment at 1 year on maintenance antiTNF-alpha therapy. Inflamm Bowel Dis. 17, 1751-1758.

5. de Miguel Criado, J., del Salto, L.G., Rivas, P.F., del Hoyo, L.F., Velasco, L.G., de las Vacas, M.I., Marco Sanz, A.G., Paradela, M.M. \& Moreno, E.F. (2012). MR imaging evaluation of perianal fistulas: spectrum of imaging features. Radiographics. 32(1), 175-194. doi: 10.1148/ rg.321115040 